TITLE:

\title{
A High-Resolution, Precipitable Water Vapor Monitoring System Using a Dense Network of GNSS Receivers
}

\section{AUTHOR(S):}

Sato, Kazutoshi; Eugenio Realini; Tsuda, Toshitaka; Oigawa, Masanori; Iwaki, Yuya; Shoji, Yoshinori; Seko, Hiromu

\section{CITATION:}

Sato, Kazutoshi ...[et al]. A High-Resolution, Precipitable Water Vapor Monitoring System Using a Dense Network of GNSS Receivers. Journal of Disaster Research 2013, 8(1): 37-47

\section{ISSUE DATE:}

2013-02

URL:

http://hdl.handle.net/2433/173916

\section{RIGHT:}

(C) 2013 Fuji Technology Press Co, . Ltd.; This is not the published version. Please cite only the published version.; この論文は出版社版で ありません。引用の際には出版社版をご確認ご利用ください。 


\title{
A High-Resolution, Precipitable Water Vapor Monitoring System Using a Dense Network of GNSS Receivers
}

\author{
Kazutoshi Sato $^{1), 2)}$, Eugenio Realini ${ }^{1)}$, Toshitaka Tsuda ${ }^{1)}$, Masanori Oigawa ${ }^{1)}$, \\ Yuya Iwaki ${ }^{1)}$, Yoshinori Shoji ${ }^{3)}$, and Hiromu Seko ${ }^{3)}$ \\ 1) Research Institute for Sustainable Humanosphere (RISH), Kyoto University \\ 2) Center for the Promotion of Interdisciplinary Education and Research, Kyoto University \\ 3) Meteorological Research Institute (MRI), Japan Meteorological Agency (JMA) \\ E-mail: sugar@ rish.kyoto-u.ac.jp, eugenio_realini@rish.kyoto-u.ac.jp, tsuda@rish.kyoto-u.ac.jp, masanori_ohigawa@rish.kyoto-u.ac.jp, \\ yuya_iwaki@rish.kyoto-u.ac.jp, yshoji@mri-jma.go.jp, hseko@mri-jma.go.jp
}

\begin{abstract}
This work describes a system aimed at the near real-time monitoring of precipitable water vapor (PWV) by means of a dense network of Global Navigation Satellite System (GNSS) receivers. These receivers are deployed with a horizontal spacing of 1-2 km around the Uji campus of Kyoto University, Japan. The PWV observed using a standard GPS meteorology technique, i.e., by using all satellites above a low elevation cutoff, is validated against radiosonde and radiometer measurements. The result is a RMS difference of about $2 \mathrm{~mm}$. A more rigorous validation is done by selecting single GPS slant delays as they pass close to the radiosonde or the radiometer measuring directions, and higher accuracy is obtained. This method also makes it possible to preserve short-term fluctuations that are lost in the standard technique due to the averaging of several slant delays. Geostatistical analysis of the PWV observations shows that they are spatially correlated within the area of interest; this confirms that such a dense network can detect inhomogeneous distributions in water vapor. The PWV horizontal resolution is improved by using high-elevation satellites only, with the aim of exploiting at best the future Quasi-Zenith Satellite System (QZSS), which will continuously provide at least one satellite close to the zenith over Japan.
\end{abstract}

Keywords: GPS meteorology, GNSS, PWV, Meteorological hazard

\section{INTRODUCTION}

The frequency and intensity of severe weather events are projected to increase due to the effects of global warming, and meteorological and hydrological hazards, such as sudden increases in river levels, floods, or landslides, may be caused. Torrential rain caused by severe thunderstorms is becoming a serious problem in urban areas because such events can develop suddenly, and it may be difficult to issue timely warnings to the public. The accurate prediction of localized heavy rain on a horizontal scale of several kilometers is still a challenging task in current mesoscale numerical prediction models, partly due to the lack of a now-casting meteorological observation network with fine spatial resolution. Information on water vapor is a particularly important factor in improving the prediction of heavy rains. Note that a weather radar can detect a cloud only after precipitation starts. It is necessary, therefore, to develop an observation system that can monitor the local inhomogeneities of water vapor distributions that could be associated with the formation of convective clouds before torrential rains occur.

The Global Navigation Satellite System (GNSS), represented by GPS, is now widely used for civilian applications, including those related to geosciences, such as the monitoring of tectonic motion, the monitoring of volcanic eruptions, and the analysis of earthquakes. The basis of the GPS is very precise measurement between the satellite and the surface receiver. If the distance between the receiver and, at least, three satellites are determined, the surface position is computed from well defined satellite position. The distance measurement is conducted by measuring the travel time of time signal emitted from the satellite and is received by surface receiver. However, the measured time is affected by various errors, either due to hardware limitations to the measurement precision, such as clock errors, or to physical phenomena that cause propagation delays to the GPS radio signal, such as atmospheric refractivity. A significant source of positioning error is the propagation delay caused by the existence of water vapor. If the receiver antenna position is known, however, this delay can be used, in turn, to estimate the accumulated water vapor along the receiver-satellite path. This accumulated water vapor is mapped onto the vertical direction to estimate the integrated amount of water vapor in a column of atmosphere, i.e. the precipitable water vapor (PWV). Thus, an interdisciplinary scientific field called GPS meteorology was initiated in the 1990s on the basis of 
the principle that “one person's noise is another's signal" [1].

Networks of GPS stations exist in many countries in the world, with spatial scales ranging from global, as in the case of the International GNSS Service (IGS) tracking network, to national or regional. As an example, the Geospatial Information Authority of Japan (GSI) is operating a nationwide GPS network, called the GPS Earth Observation Network System (GEONET), which has a mean horizontal spacing of about $20 \mathrm{~km}$. The use of GEONET-derived water vapor data to support numerical weather prediction models has been extensively tested by the Meteorological Research Institute (MRI) and the Japanese Meteorological Agency (JMA) [2-4]. The JMA has been using GEONET-derived PWV for operational mesoscale analyses since 2009 [5].

Monitoring the PWV temporal and spatial variability, especially on a local scale, is deemed to be a fundamental step in improving the predictions made by mesoscale numerical weather prediction models. Shoji et al. [6] analyzed the spatial fluctuations of water vapor detected by a GPS receiver located in the Tokyo metropolitan area before a severe rainfall. The variability of the inhomogeneous component of the estimated tropospheric delay increased about 40 minutes before the severe event. They concludes that the monitoring of the local variability of the inhomogeneous component of water vapor may prove to be useful for the now-casting of local severe rain.

Regional GPS networks, however, have inter-station distances on the order of tens of kilometers, which makes them unsuitable for the accurate detection of local fluctuations in water vapor. A densification of existing GPS networks, at least in the vicinity of urban areas, is necessary for the provision of reliable and continuous water vapor monitoring infrastructures with a sufficiently high horizontal resolution.

Seko et al. [7] employed a dense network of 75 receivers over an area of about $20 \times 20 \mathrm{~km}^{2}$ around Tsukuba, Japan to study, using a tomographic technique, the three-dimensional distribution of water vapor before and during a thunderstorm. They found good correlation between the slant water vapor detected by GPS and the PWV modeled along the same slant paths; the three-dimensional water vapor distribution estimated by tomography agreed well with the rain cloud structure observed by Doppler radar. Their results also suggest that the GPS-derived water vapor increases above a height of $1 \mathrm{~km}$ about 20 minutes before the appearance of radar echoes during thunderstorm formation; this confirms that the monitoring of water vapor on a local scale may contribute significantly to the now-casting of severe storms.
In order to exploit the maximum capability of a dense network of GPS stations, however, we need to minimize the overlap in the portions of atmosphere observed by the receivers. In conventional GPS meteorology, in fact, the delays from all available GPS satellites above an elevation angle of $5-10^{\circ}$ are averaged to estimate the single value of PWV above; therefore, the horizontal resolution of GPS-derived PWV is coarse, and local inhomogeneities tend to be significantly smoothed. Additionally, the portions of the atmosphere sensed by the GPS slant paths are irregularly distributed and change over time because of the variable satellite geometry. In this article, we propose to use only satellites at high-elevation angles in order to estimate the PWV using delays that are closer to the direction of the zenith. We expect this method to significantly improve the horizontal resolution of the PWV estimates. This strategy also aimed at evaluating the possible benefits of using the future Quasi-Zenith Satellite System (QZSS) for water vapor estimation (Figure 1). The first QZSS satellite was launched in September, 2010 by the Japan Aerospace Exploration Agency (JAXA), and three more satellites are planned to be launched in the near future. When completed, QZSS will provide at least one satellite continuously close to the zenith (i.e. with an angle of elevation greater than approximately $80^{\circ}$ ) over Japan, and this will make it more suitable satellites configuration for monitoring PWV with a high horizontal resolution.

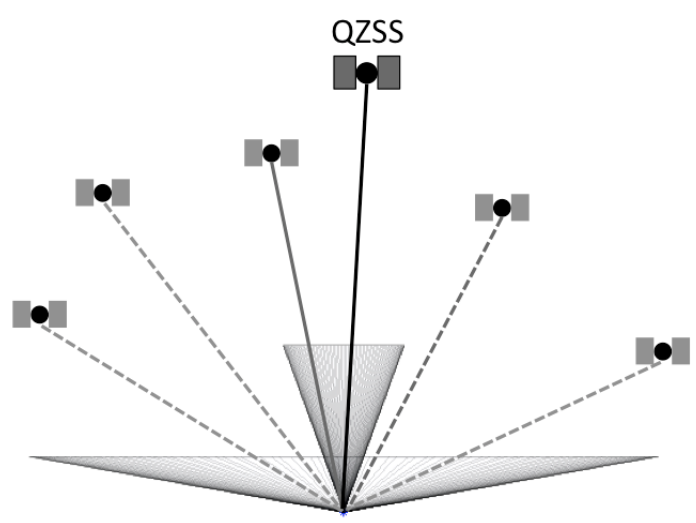

Fig. 1 Representation of the high-elevation satellite selection concept for PWV estimation, with inverse cones defined by $10^{\circ}$ and $70^{\circ}$ elevation cutoffs

Figure 2 shows a typical scenario for the daily time variation of elevation angles for GPS satellites (thin lines) and QZSS satellites (thick lines), as seen from the Uji campus of Kyoto University, Japan, where our experiments were conducted. Elevations were computed using actual orbital parameters broadcast by GPS satellites and the currently available QZSS satellite; elevations for the three future QZSS satellites 
were simulated by adding 6-hour shifts. Black lines show the elevation of the highest QZSS satellite at each epoch.

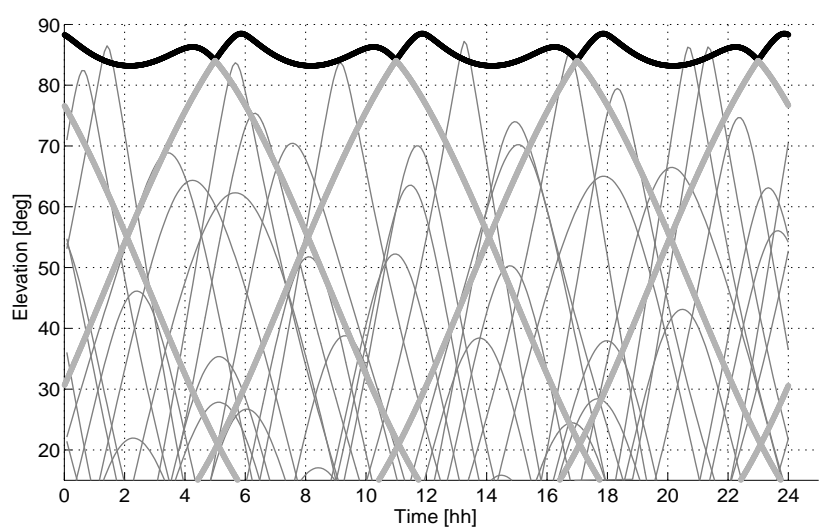

Fig. 2 Time variation of elevation angles for GPS satellites (thin lines) and QZSS satellite (thick lines, highest satellite in black) during the course of one day

A more comprehensive project that includes the current work described in this paper is the development of a near real-time system that uses a dense network of GNSS receivers to monitor high-resolution water vapor on a local scale. This article focuses on describing the system hardware and software design (section 2), the post-processing technique for GPS observations and the PWV estimation method (section 3), a validation of the estimated PWV by comparing it on radiosondes and a microwave radiometer (section 4), and the description of the proposed method to select only high-elevation satellites in order to improve the horizontal resolution of the estimated PWV (section 5). Section 6 describes the evaluation of the spatial distribution of the estimated PWV within the dense network by means of geostatistical analysis and its interpolation for producing high-resolution water vapor maps, and section 7 illustrates the possible application of this monitoring network to the implementation of a now-casting system for severe local storms.

\section{HYPER-DENSE GNSS RECEIVER NETWORK DESIGN}

We have installed a total of 17 dual-frequency GNSS stations with a horizontal spacing of 1-2 km near the Uji campus of Kyoto University, Japan (Figure 3). The stations can receive both GPS and QZSS signals, although it should be noted that during the tests described in this paper, the only currently available QZSS satellite was not yet providing precise orbital data; results including QZSS are therefore not discussed in the present paper. The stations are distributed over an area of about $10 \times 6 \mathrm{~km}^{2}$. Weather stations are installed in the same area to monitor pressure and temperature, which are needed for accurate PWV estimation. Using this dense GPS receiver network, we constructed a basic analysis system for PWV as well as a visualization system for its horizontal distribution. GPS and meteorological observations are automatically synchronized, and the data are sent from the stations to the processing servers at 5-minute intervals through wireless connections. This setup enables the construction of a near real-time monitoring system. Received data are also stored locally at each station on two separate storage devices, i.e., flash disk and SD card, to improve system redundancy. The data collection server receives observed data as binary data and converts them to 5-minute RINEX files for near real-time processing. External data needed for processing GPS observations (e.g. ephemerides, satellite status, phase center variation files, etc.) are automatically downloaded and regularly updated from the IGS FTP sites.

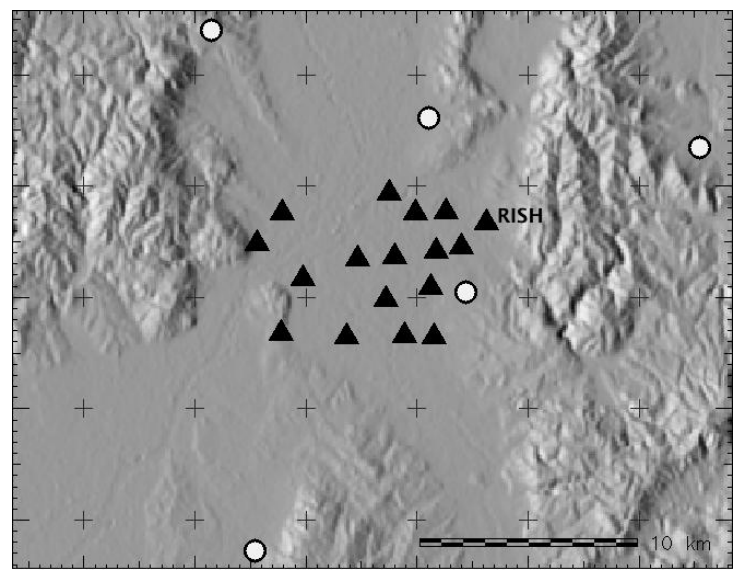

Fig. 3 The dense network of receivers installed near Uji (in Kyoto, Japan) is represented by triangles; circles indicate the location of the closest GEONET stations. The grid nodes (crosses) have a spacing of 5 $\mathrm{km}$. The shaded relief was computed using Shuttle Radar Topography Mission (SRTM3) altimetry data

In this work, we evaluate the capabilities of the PWV monitoring system under optimal conditions, that is, by post-processing dual-frequency observations and the most accurate orbits and satellite clock data provided by IGS, i.e.. final ephemerides. However, the near real-time functioning of the system would require the use the less accurate ultra-rapid ephemerides, and this would introduce additional error. We are currently testing the near real-time system performance and evaluating the use of more accurate real-time orbits and clocks, such as by using the method proposed by Shoji [8], or by using the products of the Real-Time Clock Estimation (RETICLE) system [9]. 


\section{PWV ESTIMATION METHOD}

In order to use GPS to compute the PWV, it is first necessary to estimate tropospheric delays from GPS raw data, i.e., observations.

GPS observations are essentially range between satellite and receiver derived from the accurate measurement of the signal travel time. As the GPS signal transits through the atmosphere, tropospheric refraction causes propagation delays that produce increases in the observed range. The total tropospheric delay can be divided into a hydrostatic (or dry) component, depending on the atmospheric pressure, and a wet component, which is directly related to the amount of water vapor along the satellite-receiver line-of-sight. While the dry component can be described with sufficient accuracy using current models, the wet component is rather difficult to model due to the heterogeneous distribution and the turbulent movements of water vapor. The PWV is thus estimated by including the total tropospheric delays as parameters in the GPS positioning process. A slant total delay (STD) is estimated along each satellite-receiver line-of-sight; all the estimated STDs are then mapped to the zenith direction and averaged in order to obtain the zenith total delay (ZTD). The modeled dry delay is then subtracted from the ZTD in order to obtain the wet delay along the zenith direction, from which the PWV is derived.

The results discussed in this article are obtained by post-processing dual-frequency (L1/L2) code and phase measurements with RTNet ${ }^{1}$ software, used to apply PPP (Precise Point Positioning) within a Kalman filter on GPS observations. The exact location of each GPS antenna are estimated, together with the receiver clock error and ZTD. Processing settings include an observation rate of 30 seconds, final orbits and 30-second satellite clocks provided by IGS, the L3 linear combination of L1 and L2 observations (the so-called "ionosphere-free" combination), and an elevation cutoff of $10^{\circ}$. The Global Mapping Function (GMF) [10] is used to map the slant delays in the zenith direction.

The estimated ZTD is reduced to its wet part (i.e. the zenith wet delay, or ZWD) by removing the dry component (i.e. the zenith hydrostatic delay, or ZHD), which is estimated by means of the Saastamoinen model $[11,12]$ using measured ground pressure data. The ZWD is then converted to PWV using the conversion coefficient proposed by Askne and Nordius [13]; the mean temperature of the atmosphere needed to compute the coefficient is estimated from the measured ground temperature data using empirical equations obtained from radiosonde measurements over Japan [14].

\footnotetext{
${ }^{1}$ http://www.gps-solutions.com
}

\section{VALIDATION OF THE ESTIMATED PWV}

We conducted an intensive observation campaign in July and August, 2011. The accuracy of the GPS-derived PWV was tested by comparing it with the PWV obtained from radiosondes (Vaisala RS92) and a microwave radiometer (Radiometrics MP-3000A). The GNSS station installed at the Uji Campus of Kyoto University (RISH, Figure 3) was used for the comparison, since the other instruments in its vicinity were already employed.

\subsection{Comparison with Radiosondes}

Radiosondes are sensors carried to high altitudes by balloons to measure atmospheric pressure, temperature, relative humidity, and wind velocity along their path of ascent. The amount of water vapor in each atmospheric layer can be computed from temperature and relative humidity readings as a function of altitude. The radiosonde-derived PWV in our tests corresponds to the accumulated amount of water vapor in the atmospheric layers from the ground up to an altitude of $10 \mathrm{~km}$. This threshold value was determined by analyzing the radiosonde accumulated water vapor as a function of altitude (Figure 4); $99 \%$ of the total accumulated water vapor was reached at an altitude between 8 and $9 \mathrm{~km}$ for all the radiosondes launched during this experiment. A conservative threshold value of $10 \mathrm{~km}$ was thus chosen for our tests in order to make a precise comparison between radiosonde- and GPS-derived PWV.

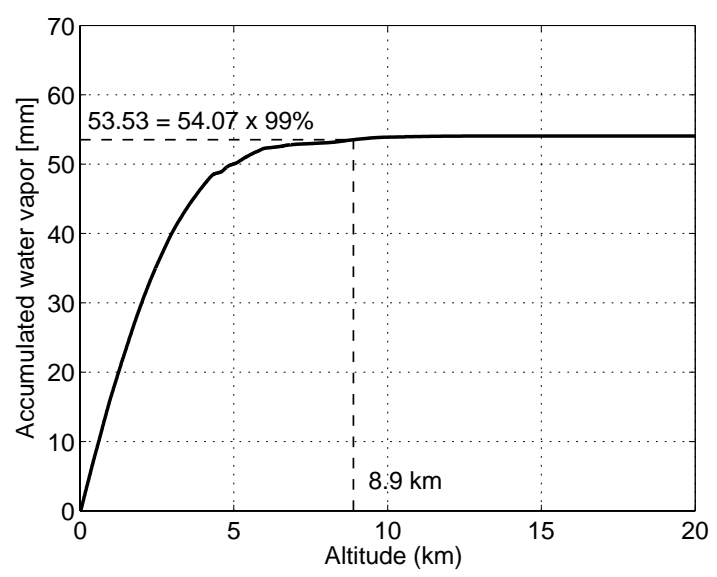

Fig. 4 Accumulated water vapor as a function of altitude (radiosonde launched on 8 August 2011 at 5:15 UTC, 14:15 local time)

During this experiment, a total of 15 radiosondes were launched; all of them drifted towards east from the launching site, which was located few hundred meters away from RISH station.

Table 1 lists the radiosonde-derived PWV, the GPS-derived PWV, and the difference between the 
two values at the moment of each radiosonde launch. The GPS-derived PWV was estimated using the standard procedure, i.e., by averaging all the slant delays from satellites above a $10^{\circ}$ elevation. Among the 15 experiments, three cases, 7/4 11:44, 7/5 3:02, and 8/12 8:46, show relatively large differences, or greater than $3 \mathrm{~mm}$. For the first two cases, each radiosonde drifted more than $20 \mathrm{~km}$ away from the launch site before reaching an altitude of $10 \mathrm{~km}$; the large difference may thus be attributed to the difference in the measured portion of atmosphere by each method. This explanation, however, could not be applied to the third case, since the drift distance was only about $9 \mathrm{~km}$. An alternative explanation is possible if we consider that radiosondes measure the amount of water vapor during their total ascent along a specific path determined by the wind direction. In our experiments, it took an average time of about 30 minutes for the radiosondes to reach an altitude of 10 $\mathrm{km}$. On the other hand, the PWV derived from GPS measurements is largely dependent on the tropospheric delay estimated along each receiver-satellite line-of-sight: depending on the satellite configuration, these may not intersect the radiosonde path (Figure 5), and they move significantly during the ascent of the radiosonde.

Table 1. Difference between radiosonde-derived and GPS-derived PWV

\begin{tabular}{|c|c|c|c|}
\hline $\begin{array}{c}\text { Launch } \\
\text { time } \\
\text { (UTC) }\end{array}$ & $\begin{array}{c}\text { Radiosonde } \\
\text { PWV } \\
{[\mathrm{mm}]}\end{array}$ & $\begin{array}{c}\text { GPS } \\
\text { PWV } \\
{[\mathrm{mm}]}\end{array}$ & $\begin{array}{c}\text { Difference } \\
{[\mathrm{mm}]}\end{array}$ \\
\hline $7 / 45: 35$ & 58.54 & 59.22 & -0.68 \\
\hline $7 / 48: 33$ & 58.44 & 59.41 & -0.97 \\
\hline $7 / 411: 44$ & 56.93 & 60.41 & -3.48 \\
\hline $7 / 53: 02$ & 32.78 & 36.74 & -3.96 \\
\hline $7 / 58: 33$ & 39.67 & 39.81 & -0.14 \\
\hline $7 / 68: 39$ & 60.33 & 63.26 & -2.93 \\
\hline $7 / 75: 42$ & 65.86 & 65.93 & -0.07 \\
\hline $7 / 79: 21$ & 65.53 & 66.48 & -0.95 \\
\hline $7 / 812: 01$ & 56.44 & 54.23 & 2.21 \\
\hline $7 / 815: 00$ & 59.53 & 58.45 & 1.08 \\
\hline $7 / 817: 48$ & 53.40 & 55.69 & -2.29 \\
\hline $8 / 125: 15$ & 54.07 & 55.71 & -1.64 \\
\hline $8 / 128: 46$ & 59.79 & 56.23 & 3.56 \\
\hline $8 / 1211: 46$ & 59.29 & 59.93 & -0.64 \\
\hline $8 / 1214: 33$ & 60.03 & 58.69 & 1.34 \\
\hline
\end{tabular}

We investigated the feasibility of employing single slant delays for direct comparison with radiosondes. Figure 6 shows the path of the radiosonde launched on $8 / 12$ at $8: 46$, in addition to the line-of-sights between the receiver and satellite 32 at the moment of the radiosonde launch (time $t$ ) and when the radiosonde reached an altitude of $10 \mathrm{~km}$ (time $t+33 \mathrm{~min}$ ). The line-of-sight was very close to the radiosonde path at time $t+33 \mathrm{~min}$. We therefore checked the difference between the accumulated water vapor measured by the radiosonde and the PWV derived only from the GPS slant delay at that time.

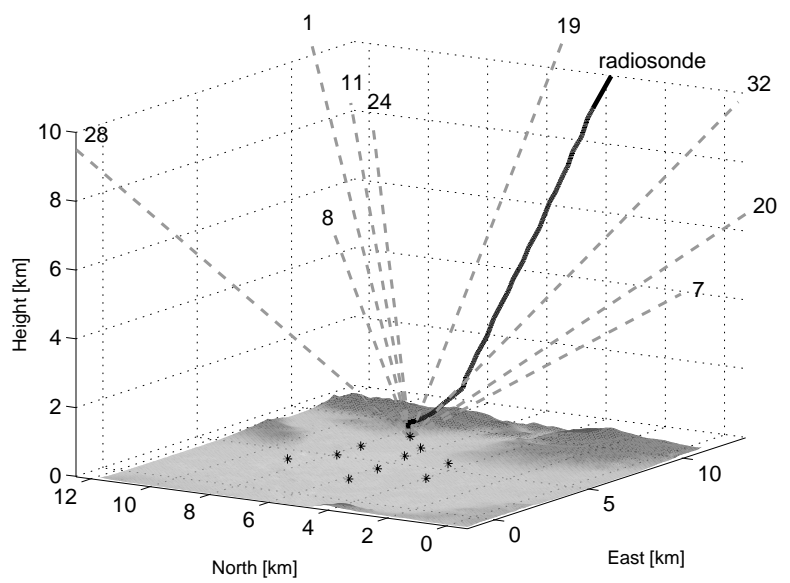

Fig. 5 The radiosonde path on 8/12 8:46 UTC (solid line), compared to the GPS satellite lines-of-sights at the moment of the radiosonde launch (dashed lines)

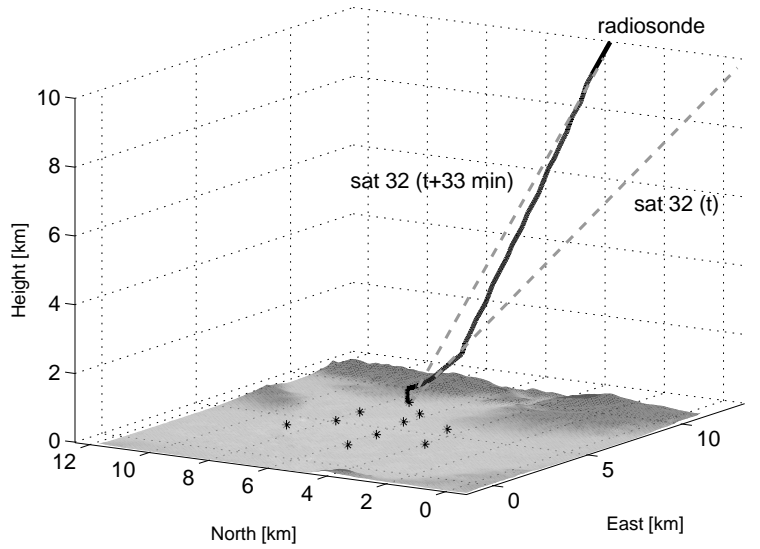

Fig. 6 The line-of-sight for GPS satellite 32 at the time of the radiosonde launch (dashed line on the right) and after 33 minutes later (dashed line on the left); the radiosonde path is represented by the solid line

Figure 7 shows the GPS-derived PWV obtained by using all satellites above $10^{\circ}$ of elevation (thick solid line) and by using only satellite 32 (dashed thin line). The cross shows the radiosonde-derived PWV, plotted at time $t+33$ min. A 5-minute moving average was applied to the GPS slant delay time series to reduce measurement noise. The GPS slant-derived PWV, though it includes some residual fluctuation which should be eliminated by averaging multiple paths, is much closer to the radiosonde-derived PWV, as expected. 


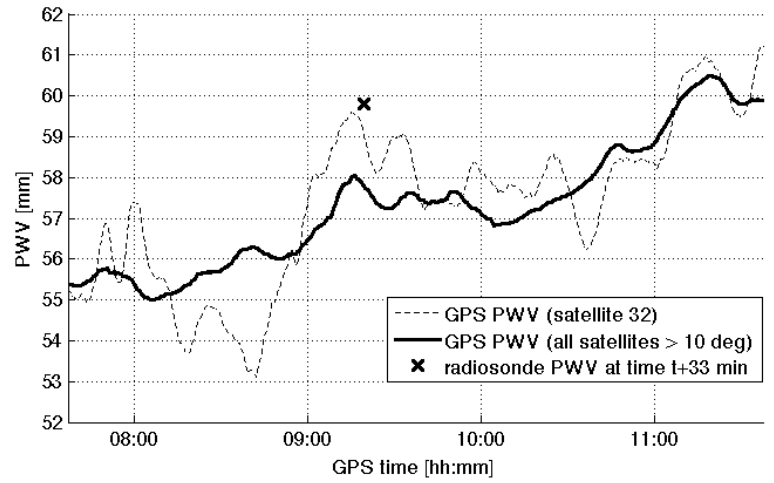

Fig. 7 Comparison between the GPS-derived PWV using all available satellites above $10^{\circ}$ (solid thick line), the PWV using only satellite 32 (dashed thin line) and the radiosonde PWV, plotted at time $\mathrm{t}+33$ minutes

This result was confirmed by a second test (radiosonde launched on 8/12 at 11:46 UTC), during which one of the GPS slant paths moved sufficiently close to the radiosonde path.

These results suggest us at least two considerations. First, a proper comparison between radiosonde and GPS for the estimation of PWV should consider the information provided by GPS slant delays, taking into account their geometry and comparing slant delays that are temporally and spatially close to the radiosonde path. Second, the PWV estimated by single GPS slant delays may be used directly for the evaluation of the amount of water vapor along specific directions. The latter consideration is particularly relevant to supporting the use of high-elevation satellites and, eventually, of QZSS to accurately monitor the PWV with high horizontal resolution (Section 6). The good accuracy of PWV derived directly from GPS slant delays was verified also in the radiometer comparison, as described in subsection 4.2.

\subsection{Comparison with a microwave radiometer}

The amount of integrated water vapor along specific directions from the ground can also be measured by microwave radiometers, which detect microwave emissions from water molecules in the atmosphere. The radiometer used in this work was installed on the roof of a building on the Uji campus of Kyoto University. It was a few meters away from the RISH station (belonging to the dense network) and provided measurements along several lines-of-sight above an elevation cutoff of $10^{\circ}$, including the zenith direction. The comparison described here was carried out by selecting the radiometer water vapor measurements along the zenith direction and computing the difference with the PWV estimated by the RISH station, during a period of about ten days, from 12
August to 21 August. The time intervals during which precipitation occurred were not included in the comparison because the high noise affects the radiometer measurements. It is worth noting that the two main advantages of using GPS measurements instead of a radiometer for PWV estimation is that GPS results are not adversely affected by rain and that the GPS technique for water vapor retrieval does not require an a priori calibration. The radiometer showed a similar level of consistency with GPS as did the radiosondes (about $2 \mathrm{~mm}$ in RMSE). Also, in this case, we performed a direct comparison by employing a single slant delay in order to verify whether the estimated PWV accuracy could be improved by selecting high-elevation satellites, or by using QZSS, rather than by averaging all visible satellites. We selected the GPS satellite 2, which reached an elevation of about $87^{\circ}$ every day during our test period. We mapped its delay in the zenith direction and computed the PWV. Also in this case, a 5-minute moving average was applied to the GPS slant delay time series. Figure 8 shows the comparison between the PWV derived from satellite 2, from all satellites above $10^{\circ}$ and from the radiometer, during a timespan when satellite 2 elevation was higher than $70^{\circ}$. The elevation of satellite 2 is plotted above the PWV graph, as a reference.
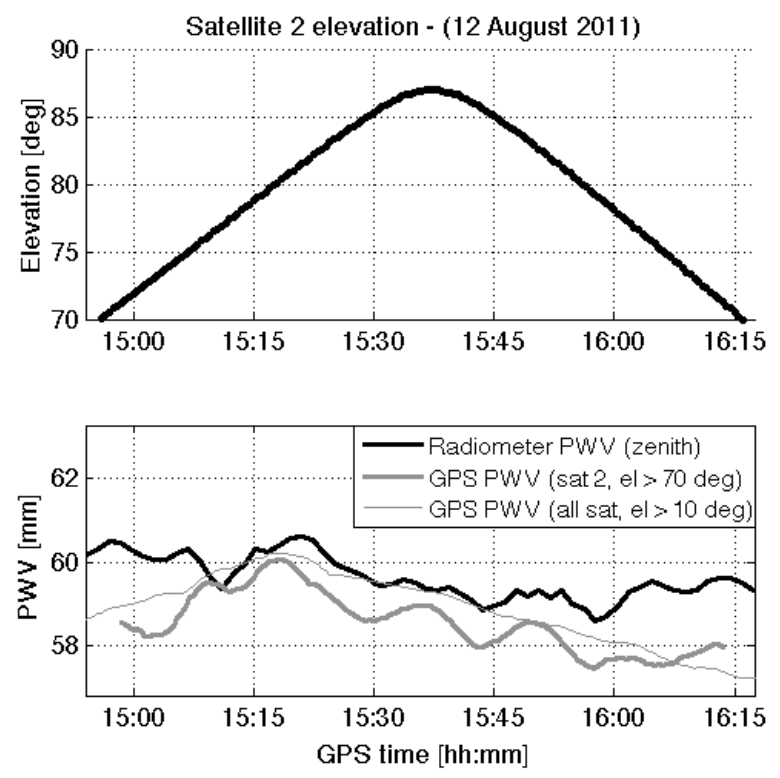

Fig. 8 Comparison between PWV time series obtained from the radiometer (black thick line), by using all GPS satellites above $10^{\circ}$ (grey thin line) and by using only satellite 2 (grey thick line)

The short period fluctuations detected by the microwave radiometer are more closely reproduced when only the tropospheric delay from the GPS satellite 2 is used. On the contrary, these fluctuations 
are essentially smoothed out when all satellites above $10^{\circ}$ are used. This is further evident that the short period fluctuations detected by single GPS slant delays are not due to measurement noise, but rather to spatial inhomogeneity on water vapor distribution. It is worth pointing out that the bias of about $1 \mathrm{~mm}$ between the radiometer and the GPS satellite 2 water vapor time series may be due either to a radiometer calibration error or to a modeling error in the GPS processing. However, given the good agreement between radiosondes and single slant GPS delays (lower than 1 $\mathrm{mm}$ ), as described in section 4.1, we are led to think that the radiometer calibration error is a more likely cause. The better agreement shown in Figure 8 between the radiometer and the PWV estimated using all satellites above $10^{\circ}$, rather than by the highest satellite only, was not evidenced during other tests.

\section{Preliminary Results fOR THE SELECTION OF HIGH-ELEVATION SATELLITES}

The standard method of using GPS processing to compute the ZTD involves the averaging of all the tropospheric delays estimated within an inverse cone defined by the elevation cutoff used for the processing of GPS observations. Using a dense network of GPS stations, such as the one used in this work, causes the averaging inverse cones to overlap significantly. Figure 4 shows that on a typical summer day in Japan about the $90 \%$ of the water vapor is in the first $5 \mathrm{~km}$ from the ground. This is under subtropical conditions, but it is probably a good approximation for tropical regions as well. Inter-station distances of about $2 \mathrm{~km}$ for the dense GPS network would be enough to get independent data when satellites above $80^{\circ}$ of elevation are selected. In this way, each receiver would observe a different portion of the atmosphere, and the overlap among averaging cones would be minimized. While using an elevation threshold of $80^{\circ}$ may not be feasible with the GPS constellation alone, since it would drastically reduce the number of epochs with available observations, it should be noted that in the near future multi-constellation positioning will be enabled by the completion of GLONASS, Compass, and Galileo global positioning systems. In a multi-constellation scenario, the availability of high elevation satellites would be significantly increased. Such a high elevation threshold would also be useful in terms of employing QZSS for water vapor estimation. When the QZSS constellation is completed, it will in fact continuously provide at least one satellite higher than about $80^{\circ}$ over Japan.

We propose to improve the horizontal resolution of the estimated ZTD by selecting only the slant delays estimated from high-elevation satellites. The procedure has to be implemented before the computation of the ZHD (section 3), and it involves the extraction of STD data from the GPS processing software output, disregarding the standard ZTD output produced by the software. STDs are then selected on the basis of the chosen satellite elevation threshold, mapped back to the zenith direction and averaged, in order to obtain a new ZTD that stems exclusively from the satellites above that specific elevation threshold. It is important to note that the overall procedure used to estimate these ZTD values involves two distinct elevation thresholds: one for the GPS processing and one for the selection of STDs. The one for the GPS processing should be kept as low as possible in order to maximize the number of observations available for the positioning process, i.e., for the tropospheric delay estimation. Figure 9 shows an example of PWV estimated by one station (RISH) on 16 October 2011 but using different elevation thresholds $\left(10^{\circ}\right.$ and $\left.60^{\circ}\right)$ to select STDs.

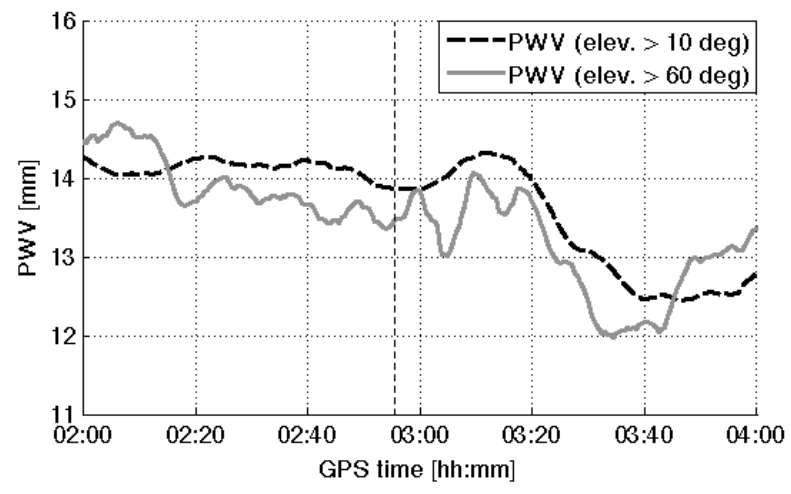

Fig. 9 Comparison between the PWV estimated by RISH station using GPS satellites above $10^{\circ}$ (black thick dashed line) and $60^{\circ}$ (grey thick solid line); the thin dashed line marks the time corresponding to the interpolated maps shown in Figures 11 an 12

\section{STATISTICAL ANALYSIS OF THE PWV SPATIAL DISTRIBUTION AND KRIGING INTERPOLATION}

Monitoring and visualizing the temporal and spatial variability of PWV over the area of interest is a crucial step in gaining a better understanding of water vapor behavior before or during severe weather events. The production of high-resolution maps and animations based on the PWV estimated by a dense GNSS network requires a reliable interpolation technique that takes full advantage of the information on the spatial variability of the water vapor. Kriging [15-17] is an unbiased and optimal method of interpolation that performs stochastic prediction on a random field, weighting observations by a function describing their 
spatial dependence, the so-called variogram ${ }^{2}$. In this work, we use the variogram for two purposes: one is to derive the interpolation weights to be used for kriging and the other is as a statistical tool for checking whether the PWV fluctuations detected within the dense network have a physical meaning (i.e. they are spatially correlated) or are just an effect of GPS measurement noise. In order to compute the variogram, the estimated PWV is modeled as the sum of a component depending on time, a component depending on station-specific biases (e.g., unmodeled antenna phase center variations and multipath), and a residual stochastic component depending on planar coordinates only. The PWV residual stochastic component is used to evaluate the empirical variogram for every timeframe of interest (an example is shown in Figure 10). The empirical variogram values are then fitted by a suitable function, such as a linear or exponential functions, in order to derive the weights for kriging interpolation.

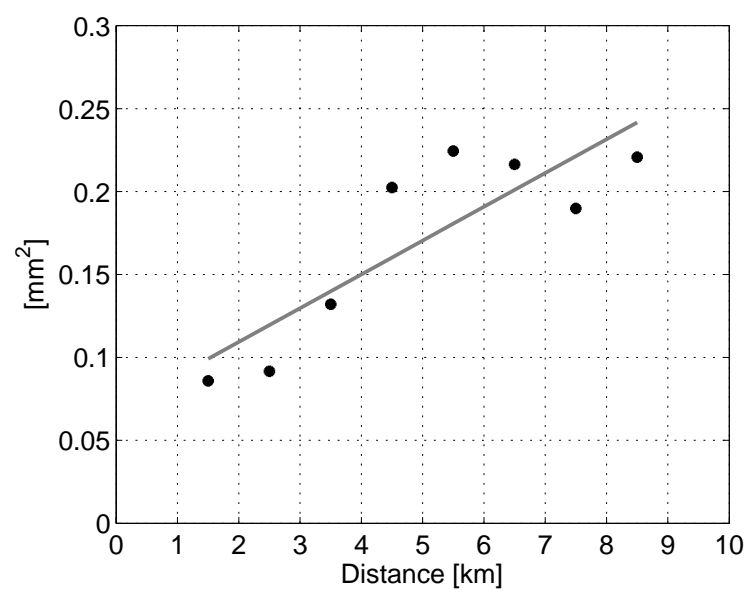

Fig. 10 Example of an empirical variogram obtained from the PWV estimated by the dense GNSS network, fitted by a linear function

Variograms obtained under average conditions of water vapor variability over the area of interest show that the PWV fluctuations estimated by the dense network on a scale of a few kilometers exhibit spatial correlation (i.e., their variability increases as a function of distance), implying that they are due not to GPS measurement noise but to a significant signal. The estimated PWV can therefore be interpolated by kriging, yielding a prediction standard deviation generally lower than $1 \mathrm{~mm}$, although in some cases the standard deviation reaches $2-3 \mathrm{~mm}$, depending on the local conditions of water vapor variability. After the

\footnotetext{
As noted by Cressie [18], the concepts of variogram and kriging, as they were introduced in geostatistics by Matheron [15], were also independently introduced in meteorology by Gandin [19], with the names of homogeneous structure function and optimum interpolation
}

application of kriging, the time-dependent and station-dependent components are added back to the interpolated PWV residuals in order to obtain maps of the total PWV distribution. Figures 11 and 12 show interpolated PWV maps obtained by using all the stations in the dense network, with the $10^{\circ}$ and $60^{\circ}$ elevation thresholds, on 16 October 2011 at 2:55:30 UTC (marked on Figure 9). The use of lower elevation thresholds produces smoother PWV, both in terms of temporal variability and of horizontal distribution. A higher level of smoothness in time and space indicates, as expected, spatial averaging over wider portions of the troposphere due to the larger inverse cones used to select slant delays.

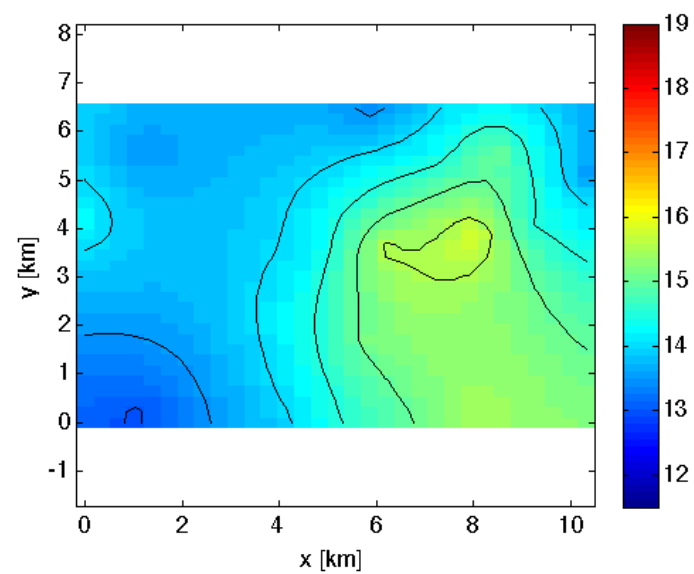

Fig. 11 PWV horizontal distribution using GPS satellites above $10^{\circ}[\mathrm{mm}]$

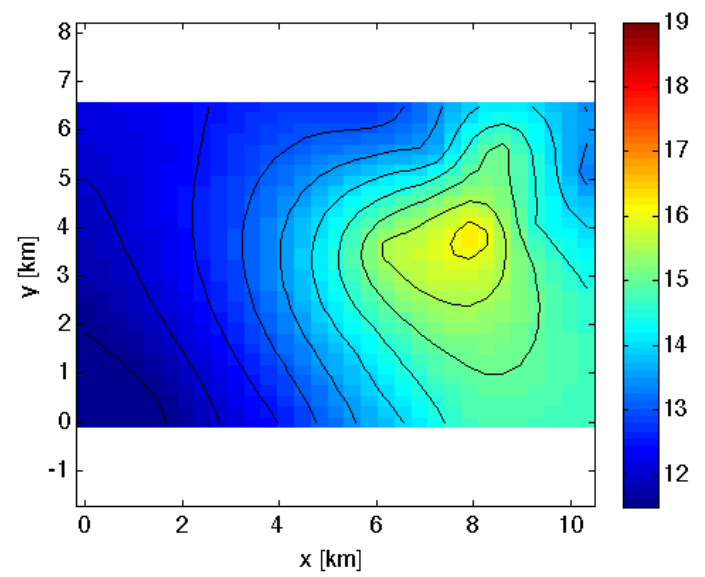

Fig. 12 PWV horizontal distribution using GPS satellites above $60^{\circ}[\mathrm{mm}]$

We conclude that the selection of high-elevation satellites shows promise for detecting water vapor fluctuations more effectively than by using the standard technique over the dense network, as well as for the production of high-resolution PWV maps. 


\section{IMPLEMENTATION CONCEPT FOR AN EARLY WARNING SYSTEM FOR NOW-CASTING SEVERE LOCAL WEATHER EVENTS}

One possible application for the dense network of GNSS receivers described in this paper is for them to serve as a water vapor monitoring network in the framework of an early warning system for now-casting local thunderstorms and torrential rain. By monitoring the temporal and spatial distribution of water vapor on a local scale, we aim to predict the occurrence of severe local rain events in the area enclosed by the network over short time spans (e.g., up to few hours). This can be particularly useful in urban areas, where the high concentration of population and economic resources increases the exposure to meteorological hazards. Nevertheless, when we apply this system to monitor wide areas, several hundreds of GNSS receivers may be needed in order to obtain a significant monitoring capability. For example, the greater Tokyo area (including Tokyo's 23 wards and the cities of Chiba, Saitama, Yokohama, and Kawasaki) covers about $1700 \mathrm{~km}^{2}$. If we undertook to deploy one receiver every $2 \mathrm{~km}$, more than 400 receivers would be needed to cover the entire area. As a reference, the greater Tokyo area currently has 10 GEONET receivers. Installing a dense network of dual-frequency GPS receivers would thus be impractical in terms of cost. Therefore, in order to keep the cost of the system down, we are evaluating the feasibility of using single-frequency receivers. However, it is necessary to compensate for the ionosphere-induced delay, which cannot be estimated with a single-frequency signal. Therefore, we are testing local ionosphere models that interpolate the ionospheric delay in order to compensate for the single-frequency measurements. Local ionospheric models to be used with a dense network of low-cost receivers can be estimated by using existing dual-frequency GNSS receivers located in the vicinity of the network (Figure 13), those belonging to existing national/regional networks, for example.

We are currently carrying out experiments employing the receivers of the Japanese nation-wide network (GEONET) to estimate the local ionosphere model, using both standard (geodetic-grade) and low-cost, single-frequency receivers for the dense network. A more detailed description of the local ionosphere modeling, the low-cost devices, and the first results is going to be published in a companion paper.

The PWV monitoring and early warning system in its current status includes the GPS observation acquisition, storage, and processing hardware as well as the software setup described in section 2. In order to achieve a functional early warning system, it is necessary to implement the real-time or near real-time processing of GPS observations and a reliable methodology for now-casting severe rain events, based on the temporal and spatial analysis of the estimated PWV. The latest water vapor maps could then be displayed, with updates every few minutes, through a web server, and severe weather warning messages could be automatically issued to users.

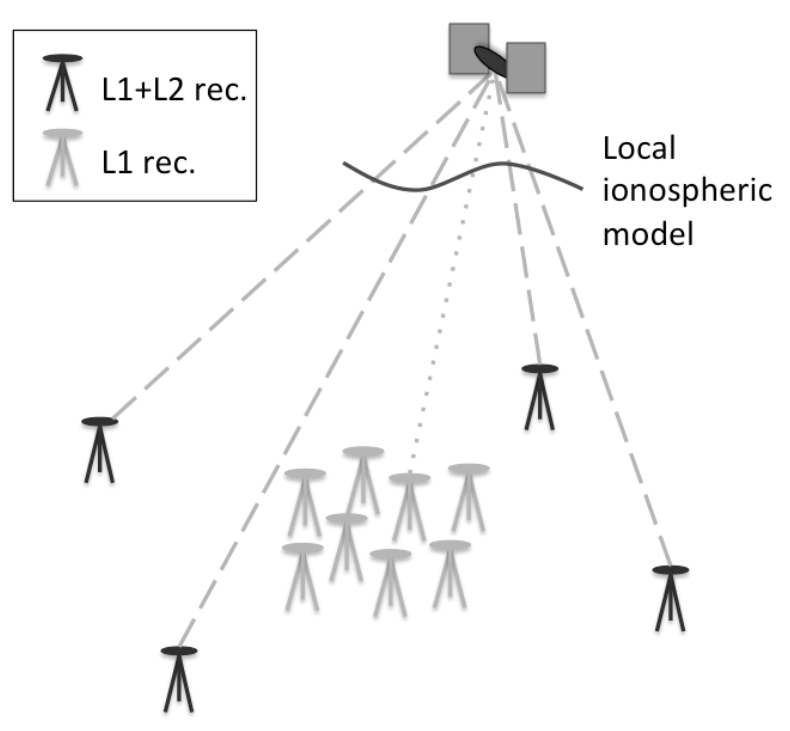

Fig. 13 Conceptual scheme for the local ionospheric model, estimated by existing dual-frequency receivers (black) and used to compensate the observations of single-frequency receivers (gray)

\section{ConClusions}

The monitoring of water vapor on a local scale is deemed to be a crucial supporting element in numerical weather prediction models, especially for the now-casting of local thunderstorms and severe rainfall events. In this paper, we have described the development and testing of a high-resolution PWV monitoring system using a dense network of GNSS receivers with inter-station distances of about $1-2 \mathrm{~km}$. The PWV estimated by the GNSS network has been validated against radiosondes and radiometer measurements, showing an RMS difference of about 2 $\mathrm{mm}$. The direct use of slant delays from a single satellite for PWV estimation improves the consistency of GPS-derived PWV with respect to radiosondes and radiometers when the satellite-receiver line-of-sight is close to their measurement direction, i.e., the balloon's path of ascent for the radiosonde and the zenith direction for the radiometer. Geostatistical analysis of the spatial distribution of the PWV estimated by the dense network exhibit spatial correlation, indicating that the estimated PWV fluctuations within the network have physical meaning. We conclude that such a dense network of GNSS receivers can be 
usefully employed for the monitoring and studying of water vapor on a local scale, and that the network tropospheric measurements have the potential to effectively support weather prediction models.

We have carried out preliminary tests involving the selection of high-elevation satellites for PWV estimation with the aim of improving the horizontal resolution of the PWV distribution. These tests were also meant to verify whether the future QZSS constellation could be effectively used in Japan to improve the monitoring of water vapor on a local scale. Preliminary results are promising, so we plan to carry out additional tests using the currently available QZSS satellite, which can provide tropospheric measurements close to the zenith continuously for about eight hours each day.

\section{Acknowledgements}

This project is supported by the coordination funds for promoting space utilization provided by MEXT. It is also supported by Sustainability/Survivability Science for a Resilient Society Adaptable to Extreme Weather Conditions (ARS), one of the Global Center of Excellence programs at Kyoto University under MEXT. We would like to thank our colleagues at RISH for their help with the radiosonde and radiometer experiments. We acknowledge the Meteorological Research Institute, Japan Meteorological Agency (MRI-JMA), for having lent us the microwave radiometer. We also thank the Board of Education of Uji city, Joyo city, Yawata city, Nagaokakyo city, Kumiyama town and Oyamazaki town, and Mr. Yoshinobu Hoso of Disaster Prevention Research Institute (DPRI), Kyoto University who cooperated with this dense observation network construction. Thanks are extended to Dr. Giovanna Venuti and Dr. Mirko Reguzzoni of Politecnico di Milano (Italy) for their insights and helpful discussions about kriging.

\section{REFERENCES:}

[1] Bevis, M., Businger, S., Herring, T.A., Rocken, C., Anthes, R.A., Ware, R.H., GPS Meteorology: Remote Sensing of Atmospheric Water Vapor Using the Global Positioning System. Journal of Geophysical Research, 97(D14), 15,787-15,801, 1992.

[2] Nakamura, H., Koizumi, K., Mannoji, N., Data assimilation of GPS precipitable water vapor into the JMA mesoscale numerical weather prediction model and its impact on rainfall forecast, Journal of the Meteorological Society of Japan, 82, 441-452, 2004.

[3] Koizumi, K., Sato, Y., Impact of GPS and TMI precipitable water data on mesoscale numerical weather prediction model forecasts, Journal of the Meteorological Society of Japan, 82, 453-457, 2004.

[4] Seko, H., Kawabata, T. , Tsuyuki, T. , Nakamura, H., Koizumi, K., Iwabuchi, T., Impacts of GPS-derived Water vapor and Radial Wind measured by Doppler radar on Numerical Prediction of Precipitation. Journal of the Meteorological Society of Japan, 82, 473-489, 2004
[5] Ishikawa, Y., The Use of Ground Based GPS Data for Meso-scale Analysis, Suuchi Yohouka Houkoku Bessatsu (Additional Volume to Report of Numerical Prediction Division), 56, 54-60, 2010 (in Japanese)

[6] Shoji, Y., Seko, H., Iwabuchi, T., Nakamura, H., A case sudy of water vapor variation in a severe thunderstorm in Tokyo by using dense network of GPS, Proceedings of International Conference on Mesoscale Convective Systems and Heavy Rain in East Asia Tokyo, 2002.

[7] Seko, H., Nakamura, H., Shoji, Y., Iwabuchi, T., The Meso- $\gamma$ scale Water Vapor Distribution Associated with a Thunderstorm Calculated from a Dense Network of GPS Receivers, Journal of the Meteorological Society of Japan, 82(1B), 569-586, 2004.

[8] Shoji, Y., A study of Near Real-time Water Vapor Analysis Using a Nationwide Dense GPS Network of Japan, Journal of the Meteorological Society of Japan, 87, 1-18, 2009.

[9] Hauschild, A., Montenbruck, O., Real-time Clock Estimation for Precise Orbit Determination of LEO-Satellites, ION GNSS 2008 Conference, Savannah, Georgia, 16-19 Sept. 2008.

[10] Boehm, J., Niell, A.E., Tregoning, P., Schuh, H., Global Mapping Functions (GMF): A new empirical mapping function based on numerical weather model data, Geoph. Res. Letters, 33, L07304, doi:10.1029/2005GL025545, 2006.

[11] Saastamoinen, J., Atmospheric correction for the troposphere and stratosphere in radio ranging satellites, in Use of artificial satellites for geodesy, Geophysics Monograph Series, 15, 247-251, 1972.

[12] Saastamoinen, J., Contribution to the theory of atmospheric refraction, Bulletin Géodésique, 107(1), 13-34, 1973.

[13] Askne, J., Nordius, H., Estimation of tropospheric delay for microwaves from surface weather data, Radio Science, 22, 379-386, 1987.

[14] Shoji, Y., PhD thesis, Kyoto University, 2010.

[15] Matheron, G., Principles of geostatistics, Economic Geology, 58, 1246-1266, 1963.

[16] Wackernagel, H., Multivariate Geostatistics: an Introduction with Applications, Springer, Berlin, 2003.

[17] Reguzzoni, M., Venuti, G., Sansò, F., The theory of general kriging, with applications to the determination of a local geoid. Geophysical Journal International, 162, 303-314, 2005.

[18] Cressie, N., The Origins of Kriging, Mathematical Geology, 22 (3), 239-252, 1990.

[19] Gandin, L.S., Objective analysis of meteorological fields: Gidrometeorologicheskoe Izdatel'stvo (GIMIZ), Leningrad, 1963 (translated by the Israel Program for Scientific Translations, Jerusalem, 1965). 